\title{
Associação entre custos por equipe de saúde da família e porte de unidade básica de saúde em uma capital do sul do Brasil
}

\author{
Association between costs per family health team and size of basic \\ health unit in a capital of southern Brazil
}

Lucas Alexandre Pedebos (https://orcid.org/0000-0001-6663-352X) ${ }^{1}$

${ }^{1}$ Gerência de Inteligência e Informação, Secretaria Municipal de Saúde de Florianópolis. Av. Prof. Henrique da Silva Fontes 6100, Trindade. $88036-700$ Florianópolis SC Brasil. lucas.enfermagem@ gmail.com

\begin{abstract}
The scope of this article is to verify the existence of the association between the number of family health teams in primary care units and the individual costs per team. A total of 46 basic health units were included in the sample to verify association through multiple linear regression between the costs per team (dependent variable) and the number of teams per unit, controlled by the number of health care procedures (independent variables). The data used were derived from a study of assessment of costing by absorption. There was an important inverse association between the size of the unit and the costs per team, controlled by the number of health care procedures (adjusted $R^{2}=0.69 ; p<0.001$ for $C I=95 \%$ ), although costs per team in similar sized units varied considerably. The results encountered tend to suggest the benefits of the allocation of a larger number of family health teams in the same unit, thereby reducing the costs per team.

Key words Primary Healthcare, Family Health, Healthcare Costs, Efficiency
\end{abstract}

Resumo O objetivo deste artigo é verificar a existência de associação entre o número de equipes de saúde da família em unidades de atenção primária (porte) e os custos por equipe individualmente. 46 unidades básicas de saúde foram incluídas na amostra para verificar associação por meio de regressão linear múltipla entre custos por equipe (variável dependente) e quantidade de equipes por unidade, controlado pelos atendimentos produzidos (variáveis independentes). Os dados utilizados foram derivados de um estudo de apuração de custeio por absorção. Houve importante associação inversa entre o porte da unidade e os custos por equipe, controlado pelos atendimentos ( $R^{2}$ ajustado $=0,69 ; p<0,001$ para IC $=95 \%$ ), ainda que os custos por equipe em unidades de mesmo porte tenham variado consideravelmente. Os resultados encontrados versam em favor da alocação de um maior número de equipes de saúde da família na mesma unidade, reduzindo os custos por equipe.

Palavras-chave Atenção Primária à Saúde, Saúde da Família, Custos de Cuidados de Saúde, Eficiência 


\section{Introdução}

A estruturação do sistema de saúde no Brasil pós Constituição Federal de 1988, que define a saúde como direito de todos e dever do Estado, vem passado por uma série de mudanças na estrutura de gestão. Aspectos administrativos fundamentais como investimento mínimo em saúde e formato de repasses entre os entes federativos ainda são importante alvo de discussão e, nos últimos dois anos, passaram novamente por modificações, sendo pouco provável que sejam as últimas dadas as ainda existentes divergências entre governo das diferentes esferas, organizações sociais e educacionais sobre esses aspectos ${ }^{1}$.

Apesar destas discussões recentes, alguns aspectos mais amplos da organização do sistema de saúde parecem estar mais consolidados. Dentre estes, a mudança de modelo organizacional anteriormente baseado em hospitais e ambulatórios especializados para o foco na atenção primária à saúde (APS) talvez tenha sido um dos mais estruturantes ${ }^{2,3}$.

Ambos as formas já foram amplamente testadas ao redor do mundo e os vários estudos desenvolvidos demonstram que um sistema de saúde baseado em APS, quando comparado ao outro modelo, é capaz de garantir respostas de qualidade aos problemas de saúde em um menor tempo, com menor custo e com melhores resultados na saúde da população $0^{4,5}$.

Contudo, a realidade brasileira não pode ser diretamente comparada à de outros países com sistemas de saúde universais por diversos motivos, dentre os quais 1) a imensidão territorial e populacional do Brasil - o único país com mais de 100 milhões de habitantes com sistema de saúde público, universal, integral e gratuito ${ }^{6,7}$; 2) a contrastante realidade da ampla diversidade socioeconômica brasileira, contendo, ao mesmo tempo, indicadores sociais e econômicos de países desenvolvidos e da África subsaariana ${ }^{8}$; e 3) a imaturidade enquanto nação, com uma democracia das mais jovens do mundo, um sistema tributário dos mais regressivos do mundo (contrastante com a intenção de prover educação e saúde à todos) e importantes conflitos políticos ${ }^{9,10}$.

Pelos dois primeiros pontos citados, o sistema anterior (gerido pelo INAMPS) sofria duras críticas de sanitaristas, sendo a gestão centralizada acusada de não conseguir entender as diversas realidades locais, principalmente das cidades mais distantes dos grandes centros urbanos e fora do eixo Sudeste-Sul. Assim, na reestruturação do sistema de saúde pós 1988 a municipa- lização passa a ser apontada como um caminho possível para que as particularidades possam ser contempladas, sendo a gestão federal responsável por diretrizes gerais enquanto a operação seria de responsabilidade principalmente dos municípios, sobretudo com relação à atenção primária ${ }^{11}$.

Assim, o sistema de saúde brasileiro, em meados da década de 1990, possuía claros direcionamentos para a gestão municipal com foco na expansão da atenção primária como porta preferencial de entrada no sistema de saúde, ainda que não tenha havido uma reforma ampla e reestruturante do sistema de saúde para isso. Aos poucos, a gestão das instituições de saúde passou a ser direcionada para os municípios, mas sem um processo direto de rediscussão dos papéis de cada ente federativo ou instituição. Todos os mais de 5.500 municípios brasileiros passavam a aprender a fazer um novo sistema de saúde envolvendo toda a sua complexidade de gestão nos mais diferentes ambientes de cuidado à saúde ${ }^{12,13}$.

Contudo, para que possamos chegar a padrões semelhantes ao de países como Inglaterra, Espanha, Portugal e Holanda, nos quais a APS realmente é ordenadora da rede de saúde, ainda há muito a caminhar. Para começar, como citado anteriormente, não é comum nos demais países com sistema de saúde universal o município ser o gestor. A conformação mais comum é aquela em que se utiliza das regiões de saúde como forma de organizar o sistema, com caráter administrativo e financiamento próprio. Não há correlato no Brasil já que as regiões de saúde aqui acabam servindo muito mais como articuladoras e pacificadoras em cenários políticos complexos de diminutos municípios que dependem dos serviços alocados fora de seus territórios. A exceção da regionalização em outros países seria a Finlândia que, ao instituir um sistema de saúde universal percebeu que o grande número de municípios e a pequena densidade demográfica da maioria destes tornaria o sistema de difícil gestão e pouco eficiente, reduzindo, então, o número de municípios, movimento contrário ao que acontece no Brasil, mesmo que aqui não costume haver base técnica que sustente a criação de novos municípios com menos de 10 mil habitantes ${ }^{14,15}$.

Ainda que o Brasil tenha optado pelo mesmo modelo focado na APS como tantos outros países com sistema universal de saúde, modelo este amplamente estudado com relação aos menores custos e benefícios em saúde quando confrontado com sistema hospitalocêntricos ${ }^{16}$, as particularidades nacionais com relação à organização da rede de saúde são muitas, como citado 
no parágrafo anterior. Por isso, a transposição de resultados de estudos internacionais com foco em eficiência da rede de atenção à saúde deve ser feita com cuidado redobrado, até porque o papel decisório em nosso país envolve a união, estados e municípios (por meio das comissões intergestores), além das instâncias de controle social.

No cenário nacional, ainda que a maioria dos municípios brasileiros seja de pequeno porte (aqueles com até 25 mil habitantes, 74,8\% do total), os municípios com mais de 100 mil habitantes (grande porte, apenas 5,5\% do total) abrigam $56,3 \%$ da população brasileira ${ }^{17}$. Dessa forma, se não se pode criar padrões nacionais pelo cenário amplamente variado, políticas que busquem gastos mais eficientes em saúde nos maiores municípios acabam por beneficiar uma grande parcela da população e, consequentemente, um montante muito importante de recursos públicos.

Dessa forma, objetiva-se nesse estudo verificar se há associação entre o porte (em número de equipes de saúde da família) de uma unidade de saúde e os custos de cada equipe.

\section{Organização da Atenção Primária em Saúde}

A atenção primária no Brasil começa ainda antes da constituição do SUS quando o debate sobre esse modelo de organização da rede de atenção à saúde ganha força mundialmente na década de 1970. Ainda sem um direcionamento claro enquanto política por parte das organizações nacionais, algumas unidades básicas de saúde constituídas por médicos clínicos gerais, ginecologistas, pediatras, técnicos de enfermagem e enfermeiros foram sendo montadas ao redor do país ${ }^{18}$. Essas unidades, ainda existentes, são conhecidas hoje como unidades do modelo tradicional.

Foi somente no início da década de 1990, com as leis orgânicas da saúde e após o novo pacto federativo instituído pela constituição, que a descentralização começa a tomar corpo com o repasse do exercício de atividades de assistência à saúde para o município. Aliado a isso, em 1994 surge o Programa de Saúde da Família (PSF) no intuito de criar um modelo organizacional e assistencial de constituição simples e grande impacto na saúde da população, passando a se chamar mais tarde de Estratégia de Saúde da Família (ESF) e sendo considerada a forma prioritária de organização da atenção primária em saúde no país ${ }^{18,19}$.

Desde o início do PSF a constituição mínima da equipe de saúde da família (EqSF) permane- ce inalterada, contando com médico generalista, enfermeiro, técnico ou auxiliar de enfermagem e agentes comunitários de saúde. Sugere-se, ainda, a associação desta a uma equipe de saúde bucal (EqSB), contanto com cirurgião dentista e auxiliar ou técnico em saúde bucal. Tal composição permanece inalterada desde o início do PSF, sendo que as revisões da Política Nacional de Atenção Básica (PNAB) trouxeram apenas alguma flexibilização quanto a carga horária dos profissionais que compõem as EqSF, sem modificar a conformação da mesma ${ }^{7,20,21}$.

Cada uma dessas EqSF deve ser possuir um território adscrito no qual se torna responsável pela população residente neste. Desde a criação do PSF a população recomendada era de 3.450 pessoas (mínimo de 2.400 e máximo de 4.500), sendo que PNAB de 2017 muda essa recomendação para uma faixa de 2.000 a 3.500 , considerando 2.000 para os cálculos de teto de equipes credenciadas e financiamento pelo Ministério da Saúde (anteriormente esse cálculo considerava as 3.450 pessoas recomendadas). A faixa de variação existe para que o gestor local possa adaptar a população adscrita considerando aspectos geográfi$\cos$, demográficos e socioeconômicos ${ }^{7,21,22}$.

Sobre a quantidade de equipes por UBS, a PNAB de 2006 trazia a sugestão de que houvesse uma unidade para cada 12 mil habitantes em conglomerados urbanos quando as equipes fossem de saúde da família (30 mil habitantes para unidades do modelo tradicional), o que daria de 3 a 4 equipes por unidade, tomando o parâmetro vigente à época de população adscrita de 3450 por $\mathrm{EqSF}^{3}$. No entanto essa sugestão deixou de ser dada nas PNABs posteriores, e com a expansão da APS nas grandes cidades UBS com mais de 4 equipes passaram a ser bastante comuns, como as clínicas da família na capital Rio de Janeiro-RJ por possível geração de economia de escala e melhor aproveitamento dos poucos espaços urbanos possíveis para construção de unidades de saúde.

\section{Economia de escala}

A economia de escala é um fenômeno econômico frequentemente observado com o aumento do ambiente de produção. É "obtida quando o custo médio dos procedimentos diminui pelo bom aproveitamento da capacidade instalada, com distribuição dos custos fixos para o maior número possível de procedimentos, eliminando ociosidades e desperdícios"23. Em outras palavras, com o aumento da produção há a diminuição do custo médio do produto/serviço produzido. 
Uma das formas em que é observada é quando há o aumento da produção de determinado bem (produto ou serviço) acima do crescimento proporcional dos custos fixos, fazendo com que o peso destes sobre os produtos/serviços seja proporcionalmente menor a cada novo produto/ serviço produzido. Outra das formas possíveis é quando a concentração do processo produtivo em determinado estabelecimento facilita a logística envolvida e reduz seus custos atribuídos a cada produto ${ }^{24}$.

No campo da saúde, alguns relatados na literatura nacional se referem ao ganho de escala por meio da constituição de redes de saúde para o compartilhamento de serviços especializados. Dessa forma, retira-se a necessidade da construção de pequenos ambulatórios especializados, muitas vezes com capacidade ociosa, para a centralização em ambulatórios maiores e mais equipados, mas com uso completo da capacidade instalada, evitando desperdício ${ }^{23,25}$.

Mas será que esse cenário de ganho de escala com o aumento do setor produtivo pode se repetir para unidades básicas de saúde, como acontece de maneira geral em ambientes de produção? Ou será que o tipo de serviço prestado em uma UBS é tão particular a ponto de contrariar essa regra? Infelizmente não se encontrou literatura nacional com este tipo de trabalho ou documento oficial do Ministério da Saúde que descreva a escolha por unidade de pequeno porte nos programas de financiamento, mas um estudo realizado pela Associação Portuguesa de Economia em Saúde (APES) ${ }^{26}$ foi o que balizou a diminuição do número de unidades na APS em favor do aumento do porte dessas na reforma ocorrida na década de 2000 no sistema de saúde português.

O principal achado nesse sentido foi de que o aumento da quantidade de pessoas adscritas por unidade reduz os custos por pessoa atendida. Em Portugal se utiliza uma quantidade fixa de usuários por equipe, já que a adscrição às equipes acontece por uma lista de pessoas, e não por território geográfico como no Brasil. Assim, um maior número de pessoas para a mesma unidade representa obrigatoriamente uma maior quantidade de equipes nesta mesma unidade. Dessa forma, pode-se fazer um paralelo desse achado no estudo português com o cenário presente nesse estudo.

\section{Caracterização do cenário de estudo}

Em dezembro de 2016 (período alvo deste estudo), Florianópolis possuía em sua rede de aten- ção primária um total de 49 UBS, somando 142 equipes, todas essas exclusivamente no modelo de saúde da família. As menores unidades possuíam apenas uma equipe e eram em número de 9 , e a maior unidade (apenas uma) possuía 8 equipes de saúde da família. Essas estavam distribuídas em 5 regiões de saúde, denominadas no município como distritos sanitários (DS), ainda que no ano de estudo gerenciassem apenas as UBS. A distribuição das unidades por porte (número de equipes de saúde da família) pode ser observada na Tabela 1.

Nota-se, portanto, que o município possuía a ampla maioria das UBS com até 3 equipes de saúde da família (74\%). Ao mesmo tempo em que a construção de unidades menores possibilita acesso mais fácil à população, isso só se faz importante em locais de baixa densidade demográfica, onde aglutinar mais equipes em uma mesma unidade implicaria percorrer distâncias consideravelmente maiores pela população. No entanto, a densidade demográfica na cidade possui importante variação, sendo muito maior nos distritos centro $\left(8.883,8 \mathrm{hab} / \mathrm{Km}^{2}\right)$ e continente $\left(8.374,7 \mathrm{hab} / \mathrm{Km}^{2}\right)$ do que nos demais (norte $=638,9 \mathrm{hab} / \mathrm{Km}^{2}$; leste $=773,8 \mathrm{hab} / \mathrm{Km}^{2}$; $\left.\mathrm{sul}=590,4 \mathrm{hab} / \mathrm{Km}^{2}\right)$. Ainda assim, as unidades até 3 equipes representam $50 \%$ e $72 \%$ nas regiões central e continental, respectivamente.

Diante da inexistência de estudos locais que direcionem se a rede de atenção primária deve crescer com aumento da quantidade de unidades ou aumento do número de equipes nas unidades já existentes, o gestor fica sem saber qual é a medida mais eficiente. No entanto, ainda que construir mais unidades possa ser uma medida para facilitar o acesso, os distritos continente e centro (principalmente) possuem um conjunto de unidades já bastante próximas entre si, o que empiricamente direciona a favor da expansão das unidades existentes.

\section{Método}

Trata-se de estudo quantitativo, analítico, desenvolvido com foco na eficiência sob a perspectiva da gestão do sistema de saúde. Para o desenvolvimento, utilizou-se a base de dados proveniente de estudo de custeio por absorção desenvolvido pela Secretaria Municipal de Saúde de Florianópolis-SC, no qual o custo final das unidades finais, incluindo as UBS, é o custo direto destas somado a uma fração do custo distribuído proveniente de unidades de administração e apoio ${ }^{27}$. 
Tabela 1. Distribuição de UBS por número de equipes de saúde e distrito sanitário, Florianópolis-SC, 2016.

\begin{tabular}{|c|c|c|c|c|c|c|c|c|c|c|c|c|}
\hline \multirow{3}{*}{ No equipes } & \multirow{2}{*}{\multicolumn{2}{|c|}{ UBS }} & \multicolumn{10}{|c|}{ Distrito } \\
\hline & & & \multicolumn{2}{|c|}{ Centro } & \multicolumn{2}{|c|}{ Continente } & \multicolumn{2}{|c|}{ Leste } & \multicolumn{2}{|c|}{ Norte } & \multicolumn{2}{|c|}{ Sul } \\
\hline & $\mathrm{n}$ & $\%$ & $\mathbf{n}$ & $\%$ & $\mathbf{n}$ & $\%$ & $\mathbf{n}$ & $\%$ & $\mathbf{n}$ & $\%$ & $\mathbf{n}$ & $\%$ \\
\hline 1 & 9 & 18 & - & - & - & - & 2 & 22 & 3 & 27 & 4 & 33 \\
\hline 2 & 14 & 29 & - & - & 4 & 36 & 2 & 22 & 5 & 45 & 3 & 25 \\
\hline 3 & 13 & 27 & 3 & 50 & 4 & 36 & 3 & 33 & - & - & 3 & 25 \\
\hline 4 & 6 & 12 & 1 & 17 & 2 & 18 & 1 & 11 & - & - & 2 & 17 \\
\hline 5 & 3 & 6 & 1 & 17 & - & - & - & - & 2 & 18 & - & - \\
\hline 6 & 2 & 4 & 1 & 17 & - & - & 1 & 11 & - & - & - & - \\
\hline 7 & 1 & 2 & - & - & 1 & 9 & - & - & - & - & - & - \\
\hline 8 & 1 & 2 & - & - & - & - & - & - & 1 & 9 & - & - \\
\hline
\end{tabular}

Fonte: Elaborado pelo autor com base nos dados do CNES, 2016.

Foram retirados os custos referentes à odontologia por não fazer parte do escopo deste trabalho tal análise.

Para o cumprimento do objetivo proposto, foi construído modelo contendo como variável independente os custos por equipe, definido como a divisão simples entre os custos por UBS e o número de equipes naquela UBS; e como variáveis dependentes o porte da UBS (número de equipes na unidade) e a produção total de atendimentos por unidade, tendo sido realizado regressão linear múltipla com as variáveis descritas. Esta última variável foi adicionada ao estudo para controle uma vez que os atendimentos geram importante custo variável relacionado a insumos, incluindo materiais de saúde (gaze, seringa, agulha, esparadrapo, dentre outros) e medicamentos. Foram realizados testes de normalidade, homoscedasticidade e multicolinearidade para validação do modelo de regressão.

Das 49 UBS, 46 foram consideradas na amostra, sendo duas excluídas já que durante o ano de estudo estas estavam funcionando em um mesmo ambiente físico, ainda que alguns dos custos estivesse desagregado; e outra por possuir expediente noturno, fugindo do horário padrão das demais unidade (8:00 - 12:00 e 13:00 - 17:00) e acrescentando custos pelo funcionamento em horário estendido.

Neste estudo foi utilizada a definição de porte de unidade básica de saúde como equivalente ao número de equipes por unidade, conforme as definições dadas pela Portaria GM/MS no 340 de 2013, uma das portarias que versa sobre o programa Requalifica $\mathrm{UBS}^{28}$.
O número de equipes de saúde bucal não é comparável às de saúde da família no município, já que as proporções entre estas vão de 1:1 no melhor cenário, até 1:4 no pior cenário, motivo pelo qual os custos das equipes de saúde bucal não foram considerados no estudo uma vez que gerariam um viés importante para o objetivo proposto. $\mathrm{O}$ ajuste foi realizado após a finalização do estudo de custos, sendo realizada a subtração da porção referente à odontologia (diretos e indiretos).

Os dados foram inicialmente organizados no software Microsoft Excel 2016 com pacote adicional de análise de dados. Os testes para definição do modelo de regressão e validação deste foram realizados no software R Studio v.1.1 utilizando o R versão 3.5.2. O software Tibco Statistica v.13 foi utilizado para geração adicional de gráficos.

O estudo não foi submetido à Comitê de Ética em Pesquisa já que utilizou apenas dados secundários retirados de bases de dados, sem qualquer contato direto com pessoas ou conjunto de dados que possam, de alguma forma, ser vinculados a indivíduos, atendendo ao disposto na Resolução 466/2012 e na Resolução 510/2016 do Conselho Nacional de Ética em Pesquisa.

\section{Resultados}

Os dados demonstram grande variação entre os custos por equipe e certa tendência de redução de custos por equipe com o incremento do porte, como pode ser observado no Gráfico 1. Havendo apenas uma unidade em cada um dos três 


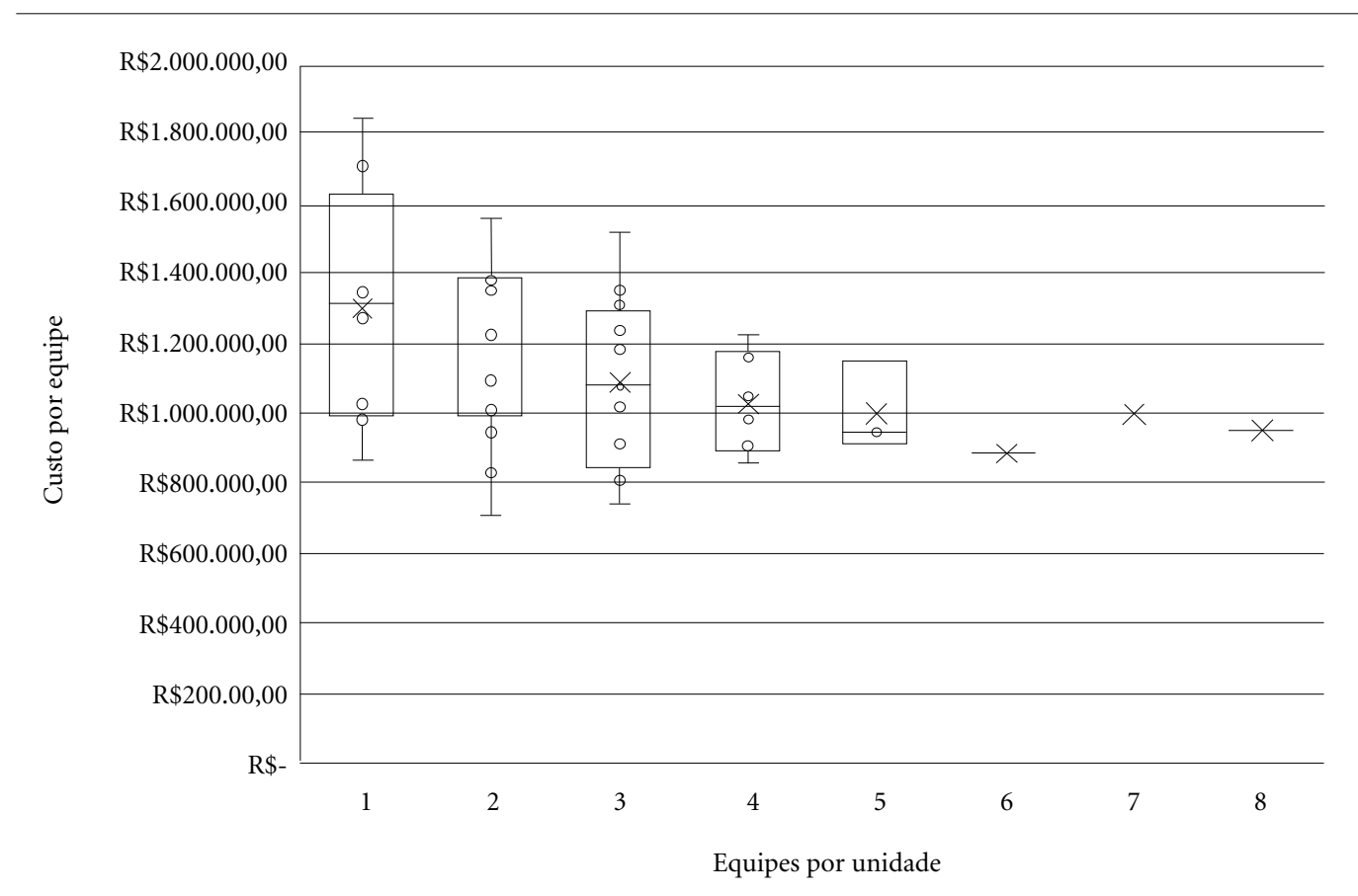

Gráfico 1. Custo por equipe de saúde vs. equipes por unidade, Florianópolis-SC, 2016.

Fonte: Elaborado pelo autor.

últimos portes consideradas na amostra (6 a 8), a avaliação descritiva dos custos nestas se mostra empobrecida com relação às demais.

Para explicar o fenômeno da variabilidade dos custos por equipe, aplicou-se o modelo de regressão linear múltipla utilizando-se como variável independente os custos por equipe, e como variáveis dependentes a quantidade de equipes por unidade (porte) e a produção geral por equipe, em número de atendimentos (incluindo atendimentos médicos, do enfermeiro e do técnico/ auxiliar de enfermagem). Há coerência teórica já que um volume maior de atendimentos deve gerar também maiores custos em alguns tipos destes, como água e materiais de consumo. Tal modelo foi o que gerou melhor poder explicativo para controle da produção por unidade. O resultado visual dessa associação pode ser observado no Gráfico 2.

A inspeção visual do Gráfico 2 demonstra importante associação entre estas variáveis. O $\mathrm{R}^{2}$ ajustado deste modelo foi de $0,69(\mathrm{p}<0,001)$, demonstrando importante associação entre as variáveis dependentes e a variável independente. Optou-se pelo indicador sintético $\mathrm{R}^{2}$ ajustado já que este penaliza o uso de variáveis em excesso no intuito de evitar a inclusão de variáveis que contribuam pouco para a explicação do fenômeno e costuma ser sugerida como medida importante nos casos em que é realizada regressão linear múltipla ${ }^{29}$. Os dados estatísticos do modelo escolhido podem ser observados na Tabela 2 .

Os testes de Shapiro-Wilk e Kolmogorov-Smirnov demonstram que a amostra provém de uma distribuição normal. O teste de Breusch Pagan demonstra homoscedasticidade dos resíduos. O teste VIF (Variable Inflation Factors) não demonstra multicolinearidade entre as variáveis independentes.

Os dados descritos demonstram que o modelo escolhido parece ter coerência com o cenário analisado, sendo que aproximadamente $70 \%$ da distribuição de custos pode ser explicada pelas duas variáveis independentes escolhidas. A escolha de modelos alternativos a este considerando a utilização da produção por categoria profissional (isoladas ou associadas dois a dois), por quantidade geral de profissionais por unidade, por custos de medicamentos ou por custos fixos (o que possui associação na literatura, pela distribuição 
Gráfico 2. Custos por equipe de saúde vs. equipes por unidade e atendimentos por equipe.

Fonte: Elaborado pelo autor.

Tabela 2. Estatística do modelo de regressão linear "custos por equipe de saúde vs. equipes por unidade e atendimentos por equipe".

\begin{tabular}{lrrr}
\hline \multirow{2}{*}{ Predictors } & \multicolumn{3}{c}{$\mathbf{y}$} \\
\cline { 2 - 4 } & Estimates & \multicolumn{1}{c}{$\mathbf{C I}$} & $\mathbf{p}$ \\
\hline (Intercept) & 614881.16 & $442693.31-$ & $<0.001$ \\
& & 787069.02 & \\
Equipes & -48502.49 & $-74770.49-$ & 0.001 \\
& & -22234.50 & \\
Atendimentos & 78.23 & $61.11-95.35$ & $<0.001$ \\
Observations & & & 46 \\
$\mathrm{R}^{2} /$ adjusted $\mathrm{R}^{2}$ & & \multicolumn{3}{c}{$0.700 / 0.686$} \\
\hline
\end{tabular}

Fonte: Elaborado pelo autor.

com parcelas menores em ambientes de produção menores, normalmente) não demonstrou associação melhor do que a encontrada no modelo selecionado.

\section{Discussão}

Os testes de regressão demonstram associação reversa importante entre os custos por equipe e o porte da unidade, quando controlado pela produção geral em número de atendimentos por equipe. Dessa forma, afirma-se a partir dos dados levantados que no município estudado é mais interessante economicamente a construção de unidades maiores, preferencialmente com 5 equipes ou mais. Não se pode afirmar que unidades com 6 ou mais equipes tenham um menor custo por equipe uma vez que na amostra utilizada o número de unidades deste porte foi bastante pequeno para que se possa realizar uma inferência favorável a estas. Outra limitação do estudo é que os dados representam apenas um ano, sendo importante que estudos desse tipo sejam realizados em outros anos para reafirmar (ou contrariar) os resultados aqui encontrados.

Observa-se neste mesmo município que a ampla maioria das UBS (74\% do total e 73\% das unidades consideradas na amostra) era composta por até 3 equipes no período estudado, 
contrariando, em aspectos econômicos achados neste estudo, a melhor distribuição de equipes por unidade. Ainda que a aglutinação de grande quantidade de equipes em uma mesma unidade possa dificultar o acesso da população aos serviços, como citado anteriormente, entende-se que a recomendação de unidades maiores poderia ser adotada pelo menos nos locais de maior densidade demográfica, como as regiões continental e central da cidade (Distritos Sanitários Continente e Centro, respectivamente), como demonstrado na Tabela 3.

Tomando a mediana de custos por equipe de unidades de 1 equipe ( $R \$ 1.312 .026,25)$ e comparando com a mediana das unidades de 4 equipes ( $\mathrm{R} \$ 1.017 .185,88)$, ambas descritas no quadro 4.1, pode-se supor que uma mudança na distribuição das equipes no município considerando a aglutinação das oito unidades menores ( 1 equipe) para a formação de duas maiores (4 equipes) geraria uma economia aos cofres públicos da ordem de $\mathrm{R} \$ 2.358 .722,96$ anuais. Tal valor seria suficiente para financiar mais uma unidade de duas equipes (mediana por equipe de R $\$ 1.164 .414,70$ ). Optou-se por considerar a mediana por ser normalmente a medida de síntese utilizada em cenários de grande variação ${ }^{29}$.

Indo mais além e pensando em uma reestruturação completa da rede para formar unidades de 5 equipes, as quais foram demonstradas como as com menor custo por equipe (evitando as inferências em unidades maiores do que estas pelos motivos citados anteriormente), temos o descrito na Tabela 3. Considerando os valores descritos na referida tabela, a economia seria suficiente para manter mais 4 unidades de 4 equipes com um acréscimo de apenas $1,3 \%$ ( $\mathrm{R} \$ 16.274 .974,09)$, totalizando 16 novas equipes; ou, mantendo como unidade padrão as de 5 equipes, 3 novas unidades desse porte com uma economia de $\mathrm{R} \$$ $1,914,102.24$ anuais, totalizando 15 novas equipes.
A economia seria ainda maior considerando questões não levantadas nesse estudo, mas que seriam observadas no mundo real. Dentre estas estão os menores gastos com logística, uma vez que a distribuição de materiais ocorreria para um menor número de unidades, otimizando o deslocamento, carga e descarga dos veículos, reduzindo custos com veículos e motoristas ${ }^{24}$.

No entanto, cabe destacar novamente que o município em estudo possui densidade demográfica bastante baixa fora da região central e continental da cidade. Assim, sendo um dos pilares fundamentais da atenção primária em saúde o acesso, a escolha entre construir unidades maiores e mais espaçadas ou menores e mais distribuídas deve considerar não apenas os custos, mas também a facilidade com que as pessoas conseguiriam chegar a tais unidades. Contudo, a maior eficiência do serviço proveniente do aumento do número de equipes na região continental, a qual possui conurbação urbana com outros dois municípios, abre margem para a discussão da regionalização, mesmo que na APS.

Se o processo de descentralização da gestão dos serviços públicos possibilitou ampliação das redes de saúde e melhor acesso aos serviços, não é verdade que as "fórmulas universais" deixaram de existir, inclusive evidenciadas pelos modelos de financiamento possíveis junto ao governo federal. Como destacado por Viana et al. ${ }^{30}$ : "A experiência internacional mostra que sistemas abrangentes são compostos de modelos variados de acesso em APS ou diversas modalidades de provisão de serviços em APS". Paim ${ }^{31}$ ainda destaca que não fazer muito sentido pensar em um modelo único para o SUS dada as distintas realidades nas diferentes cidades e regiões do país.

Para além do modelo estatístico utilizados neste estudo, os resultados encontrados corroboram com achados em estudos semelhantes realizados em outros países, no qual parece haver

Tabela 3. Economia anual com reestruturação da rede para unidades de 5 equipes.

\begin{tabular}{lcccc}
\hline no equipes & $\mathbf{n}^{\mathbf{0}}$ unidades & $\begin{array}{c}\text { Mediana de custos por } \\
\text { equipe }\end{array}$ & $\begin{array}{c}\text { Diferença por equipe } \\
\text { na reestruturação }\end{array}$ & $\begin{array}{c}\text { Subtotal (diferença x } \\
\text { equipes } \mathbf{x} \text { unidades) }\end{array}$ \\
\hline 1 & 8 & $\mathrm{R} \$ 1.312 .026,25$ & $\mathrm{R} \$ 369.414,84$ & $\mathrm{R} \$ 2.955 .318,71$ \\
2 & 14 & $\mathrm{R} \$ 1.164 .414,70$ & $\mathrm{R} \$ 221.803,29$ & $\mathrm{R} \$ 6.210 .492,01$ \\
3 & 12 & $\mathrm{R} \$ 1.084 .213,51$ & $\mathrm{R} \$ 141.602,10$ & $\mathrm{R} \$ 5.097 .675,56$ \\
4 & 6 & $\mathrm{R} \$ 1.017 .185,88$ & $\mathrm{R} \$ 74.574,47$ & $\mathrm{R} \$ 1.789 .787,18$ \\
5 & 3 & $\mathrm{R} \$ 942.611,41$ & & - \\
Total & & & & $\mathrm{R} \$ 16.053 .273,47$ \\
\hline
\end{tabular}

Fonte: Elaborado pelo autor. 
ganho de escala na construção de unidades com mais equipes, o qual foi base para a mudança nas unidades de APS em Portugal ${ }^{26,32}$. Ainda que mais estudos desse tipo sejam necessários e os documentos portugueses encontrados não citem detalhadamente a metodologia utilizada, é importante que os resultados do presente estudo não sejam contraditórios ao estudo português uma vez há semelhanças importantes entre a APS dos dois países.

Dito isto, cabe destacar que o Programa Requalifica UBS, por meio do qual o Ministério da Saúde repassa incentivos financeiros aos municípios (ou Distrito Federal - DF) para a construção de unidades básicas de saúde, prevê a construção de unidades apenas até porte $4^{28}$. Ou seja, a construção de unidades maiores do que aquelas compatíveis com 4 equipes não é estimulada financeiramente, direcionamento contrário aos achados no estudo português supracitado e no presente estudo, sob a perspectiva da eficiência.

\section{Considerações finais}

Devido a características singulares da organização da rede de atenção à saúde no Brasil, incluindo o papel de cada ente federativo, não se pode sempre transpor diretamente estudos e modelos utilizados em outros países para estruturar a conformação de unidades de saúde no nosso país. Por isso, é fundamental que estudos sejam conduzidos localmente, incluindo os de aspectos econômicos, para garantir que os serviços estejam disponíveis aos cidadãos a custos que o país possa arcar.

Sendo a atenção primária o modelo preferencial de entrada na rede de atenção à saúde, e a saúde da família a estratégia adotada para a organização da APS no país é premente a discussão de eficiência neste setor. Ainda que o país careça de maior cobertura da ESF, há que se garantir um melhor aproveitamento da capacidade instalada e minimização de desperdícios para que haja efetivo acesso da população a estes serviços.

O presente estudo apresenta uma importante associação entre custos por equipe e porte de UBS que visa auxiliar os gestores municipais na organização da APS local. Unidades maiores parecem ter um custo menor por equipe do que aquelas que comportam uma pequena quantidade de equipes. É importante frisar que a constituição de uma rede de atenção primária deve, também, considerar a facilidade com que as pessoas conseguem se deslocar para as unidades de saúde, já que o acesso é um dos pilares da APS.

No entanto não se deve pensar em uma rede de saúde sem que a eficiência dos serviços esteja em foco. Como demonstrado tanto no presente estudo quando no estudo português citado, utilizar conformações nas quais o custo por equipe é menor possibilita que mais equipes sejam constituídas, gerando a produção de maior quantidade de serviços de saúde para a população; ou utilizando essa diferença de custos para investimento em outros serviços que sejam mais necessários. 


\section{Referências}

1. Brasil. Portaria GM/MS n ${ }^{\circ}$ 3.992. de 28 de dezembro de 2017. Altera a Portaria de Consolidação n 6 GM/ MS, de 28 de setembro de 2017, para dispor sobre o financiamento e a transferência dos recursos federais para as ações e os serviços públicos de saúde do Sistema Único de Saúde. Diário Oficial da União 2017; $28 \mathrm{dez}$.

2. Conselho Nacional de Secretários de Saúde (CONASS). Sistema Único de Saúde. Brasília: CONASS; 2007.

3. Brasil. Ministério da Saúde (MS). Política Nacional de Atenção Básica. Brasilia: MS; 2006.

4. Aldulaimi S, Mora F. A Primary Care System to Improve Health Care Efficiency: Lessons from Ecuador. J Am Board Fam Med 2017; 30:3.

5. Starfield B, Shi L, Macinko J. Contribution of Primary Care to Health Systems and Health. Milbank Q 2005; 83(3):457-502.

6. Campos G. Tratado de Saúde Coletiva. São Paulo: Hucitec; 2012.

7. Brasil. Ministério da Saúde (MS). Política Nacional de Atenção Básica. Brasília: Ministério da Saúde; 2012.

8. United Nations Development Program (UNDP). Human Development Report 2016. Nova York: United Nations; 2016.

9. Ribeiro L. Democracia e segregação urbana: reflexões sobre a relação entre cidade e cidadania na sociedade brasileira. EURE 2003; 29(88):79-95.

10. Ugá M, Santos I. Uma análise da progressividade do financiamento do Sistema Único de Saúde (SUS). Cad Saude Publica 2016; 22(8):1597-1609.

11. Teixeira C. Municipalização da Saúde - os caminhos do labirinto. Rev Bras Enferm 1991; 44(1):10-15.

12. Machado J, Cotta R, Soares J. Reflexões sobre o processo de municipalização das políticas de saúde: a questão da descontinuidade político-administrativa. Interface (Botucatu) 2015; 19(5):159-170.

13. Arretche M, Marques E. Municipalização da saúde no Brasil: diferença regionais, poder do voto e estratégias de governo. Cien Saude Colet 2002; 7(3):455-479.

14. Giovanella L, Mendonça M. Atenção Primária à Saúde. In: Giovanella L, editora. Políticas e Sistema de Saúde no Brasil e Sistema de Saúde no Brasil. Rio de Janeiro: Fiocruz; 2009.

15. Teperi J, Porter ME, Vuorenkoski L, Baron JF. The Finnish Health Case System: A Value-Based Perspective. Helsinki: SITRA; 2009.

16. Friedberg M, Hussey P, Schneider E. Primary Care: A Critical Review of The Evidence on Quality and Costs of Health Care. Heal Aff 2010; 29(5):776-772.

17. Instituto Brasileiro de Geografia e Estatística (IBGE). Estimativas populacionais para os municípios e para as Unidades da Federação brasileiros para 2016 [Internet]. Disponível em: https://ww2.ibge.gov.br/home/estatistica/populacao/estimativa2016/estimativa_dou.shtm.

18. Castro A, Machado C. A política de atenção primária à saúde no Brasil: notas sobre a regulação e o financiamento federal. Cad Saude Publica. 2010; 26(4):693705.

19. Escorel S, Giovanella L, Mendonça M, Senna M. O Programa de Saúde da Família e a construção de um novo modelo de atenção básica no Brasil. Rev Panam Salud Publica 2007; 21(2):164-176.
20. Bodstein R. Atenção Básica na agenda da saúde. Cien Saude Colet 2012; 7(3):401-412.

21. Brasil. Portaria GM/MS n ${ }^{\circ}$ 2.436, de 21 de setembro de 2017. Aprova a Política Nacional de Atenção Bási$\mathrm{ca}$, estabelecendo a revisão de diretrizes para a organização da Atenção Básica, no âmbito do Sistema Único de Saúde (SUS). Diário Oficial da União 2017; 21 Set.

22. Brasil. Ministério da Saúde (MS). Universidade Federal da Bahia (UFBA). O Programa de Saúde da Família: Evolução de Sua Implantação no Brasil - Relatório Final. Salvador: UFBA; 2002.

23. Silva S. Organização das redes regionalizadas e integradas de atenção à saúde: desafios do Sistema Único de Saúde (Brasil). Cien Saude Colet 2011; 16(6):27532762.

24. Faria L. Gestão Logística Na Cadeia de Suprimentos. $4^{\mathrm{a}}$ ed. Porto Alegre: AMGH; 2014.

25. Neves L, Ribeiro J. Consórcios de saúde: estudo de caso exitoso. Cad Saude Publica 2006; 22(10):22072217.

26. Associação Portuguesa de Economia da Saúde (APES). Análise dos Custos dos Centros de Saúde e do Regime Remuneratório Experimental. Lisboa: APES; 2007.

27. Castro JD. A Utilização do Sistema de Custeio por Absorção para Avaliar Custos da Atenção Básica de Saúde: reformulações e aprimoramentos metodológicos [tese]. Campinas: UNICAMP; 2000.

28. Brasil. Portaria GM/MS n 340 , de 4 de março de 2013. Redefine o Componente Construção do Programa de Requalificação de Unidades Básicas de Saúde (UBS). Diário Oficial da União 2013; 4 mar.

29. Hair Jr J. Análise Multivariada de Dados. $6^{\mathrm{a}}$ ed. Porto Alegre: Bookman; 2006.

30. Viana A, Bousquat A, Melo G, Negri Filho A, Medina M. Regionalização e Redes de Saúde. Cien Saude Colet 2002; 23(6):1791-1798.

31. Paim J. Modelos de Atenção à Saúde no Brasil. In: Giovanella L, Lobato L, Norinha J, Carvalho A, editores. Políticas e Sistema de Saúde no Brasil. 2a ed. Rio de Janeiro: Fiocruz; 2012. p. 459-491.

32. Pisco L. Reforma da Atenção Primária em Portugal em duplo movimento: unidades assistenciais autónomas de saúde familiar e gestão em agrupamentos de Centros de Saúde. Cien Saude Colet 2011; 16(6):28412852.

Artigo apresentado em 21/04/2019

Aprovado em 02/07/2019

Versão final apresentada em 04/07/2019

Editores-chefes: Romeu Gomes, Antônio Augusto Moura da Silva 